\title{
Quatre semaines sans plastique
}

\section{Eva Mell}

Rédactrice en cheffe adjointe du Bulletin des médecins suisses

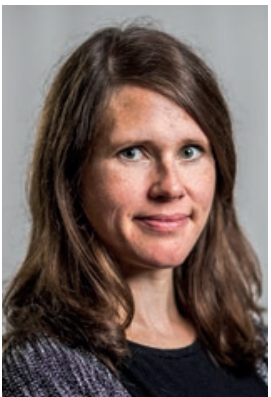

Faire mes achats pendant quatre semaines en évitant les emballages plastiques, tel est le défi personnel que je me suis fixé. Mais pour quelles raisons? Le plastique pollue et se retrouve dans les estomacs des animaux marins, qui en meurent. De plus, il se décompose en microparticules que nous ingérons ensuite via la nourriture. J'en ai mal au ventre rien que d'y penser. C'est pourquoi nous avons décidé, en famille, de renoncer au plastique pendant un mois pour commencer afin de réfléchir à nos habitudes et découvrir d'autres solutions. Peut-être arriverons-nous à instaurer de nouvelles habitudes et à nous libérer du plastique. La règle du jeu est la suivante: pendant un mois, il ne faut rien acheter qui contient ou est emballé dans du plastique, mais on peut utiliser ce dont on dispose déjà. En écrivant cela, je vous imagine déjà sourire et me conseiller d'effectuer tous les achats idoines avant de commencer le défi. C'est du moins ce que m'ont conseillé la plupart des personnes à qui j'en ai parlé. Et j'avoue que ce n'est pas un hasard si juste avant de débuter cette expérience, j'ai acheté un nouveau paquet de papier toilette dans un emballage plastique alors qu'il m'en restait encore.

J'ai aussi déjà voulu m'y frotter à l'avance en cherchant à acheter du fromage non emballé, dont j'avais déjà repéré trois sortes au rayon fromagerie du supermarché à proximité. Or, toutes les meules sont recouvertes de film plastique. Après m'avoir coupé le morceau que

La règle du jeu est la suivante: pendant un mois, nous ne pouvons rien acheter qui contient du plastique ou qui est emballé dans du plastique.

j'avais choisi, la vendeuse a jeté le film plastique qui recouvrait la meule pour le remplacer. C'est donc bien moi la responsable de cette surutilisation de plastique, mais rien ne sert de culpabiliser. En revanche, la question de savoir comment notre famille va survivre à cette nouvelle expérience culinaire reste ouverte. Nous avons néanmoins décidé dès le début de faire une exception pour les produits médicaux. Qu'il s'agisse de dentifrice, de sparadraps ou de médicaments, nous continuerons de choisir les produits les plus adéquats et non ceux dont l'emballage est le plus écologique. En tant que rédactrice d'une revue médicale et personne en bonne santé, on pourrait penser que je génère peu de déchets plastiques liés à des produits médicaux, mais la réalité est bien différente, surtout en période de pandémie. J'utilise des masques de protection jetables en matière synthétique, emballés dans des pochettes en plastique.

Nous avons décidé de faire une exception: nous continuerons d'acheter les produits médicaux les plus adéquats et non les plus écologiques.

Même hors pandémie, j'ai quand même une petite montagne de déchets liés à des problèmes plus ou moins médicaux. Elle est constituée de nombreux produits auxquels je ne peux pas renoncer (tubes de dentifrice) ou de médicaments que j'utilise moins que prévu (emballages plastiques contenant des cachets de Paracétamol et d'Ibuprofène périmés). En tant qu'utilisatrice de ces produits, il m'est difficile de procéder à des changements fondamentaux dans ce domaine. En matière d'alimentation, c'est différent. Si je change de comportement dans ma consommation alimentaire, voire renonce complètement à certains produits, je contribue, dans le meilleur des cas, à modifier l'offre. Or, pour les produits médicaux, ce n'est pas si simple, car je veux le meilleur dentifrice pour mes dents, et si je devais tomber malade, je ne voudrais pas renoncer au médicament le plus efficace au prétexte qu'il est emballé dans du plastique. Ironie du sort, cette intransigeance en matière de santé contribue à polluer l'environnement et, au final, à me rendre malade aussi.

Mais avant de sombrer dans les préoccupations au sujet des déchets plastiques dans le domaine de la santé, j'ai décidé de relever mon défi que j'espère réaliste: acheter du fromage sans emballage, car il est plus facile de relever les autres défis l'estomac plein. 